\title{
Study of Very Low Frequency Signal by Independent Component Analysis Observed Form DEMETER Satellite
}

\author{
${ }^{1}$ Shivali Verma, ${ }^{2}$ Deepak K Sondhiya,${ }^{3}$ A. K. Gwal \\ ${ }^{1,2,3}$ Space Science Laboratory, Department of Physics Barkatullah University, Bhopal-462026, India \\ Shivali.atre@gmail.com
}

\begin{abstract}
Very low frequency signals (VLF) which are observed by DEMETER satellite is often corrupted by atmospheric and manmade noise. These noises affect the quality of the VLF signal. So for quality improvement denoising of VLF signal is must. The denoising methods which are used for VLF signal often consider noise nature as Gaussian. But the nature of atmospheric and manmade noise is non-gaussian. Due to this fact denoising methods which are considering noise nature as Gaussian is not effective for the denoising of VLF signals. So in the present work we applied a statistical signal processing method for the denoising of noise being non-Gaussian is known as the independent component analysis (ICA) in which signal and noise considered to be independent and non-Gaussian.ICA based preprocesses boost the higher order correlation (bispectrum).This study also shows improvement in the signal to noise ratio (SNR) and visualized the suppressed frequency of VLF signals in the spectrogram.
\end{abstract}

Keywords - Denoise, Gaussian,Independent componemt analysis

\section{Introduction}

The analysis of radio of Very Low Frequencies (VLF) (2-20 kHz)generated by lighting discharge, earthquake, volcanic eruptions, dust storm, tornadoes, nuclear explosions or manmade transmitters[1], is useful for the study of dynamics of the Earth's ionosphere and magnetosphere as well as subterranean imagine, global communications and navigation[2].This signal is classified on the basis of their spectral characteristics[37] .Visualization and identification of spectral characteristic is possible through dynamic spectral analysis (spectrogram) of the VLF signals. Unfortunately the presences of the noise in VLF data distort the characteristics of the signal resulting in poor quality of any subsequent processing. This noise content is generated by the ionosphere because it is affected by various geophysical phenomena such as solar activities, earthquake, volcanic eruption, due to manmade noise and the surrounding of data producing system itself. Many scientists reported that few days before the earthquake occurs, noise content in VLF signal increases[3],[4]due to which signal to noise ratio(the ratio of the VLF signal to the background noise) of VLF signals significantly gets depressed. On the other side in VLF signals the man made noise is originated by human technologies and it is found that these noises are strongly dependent on the distance from the sources (power lines, radio, TV communication installations and other [5].

In this work we have studied Very low frequency (VLF) observed by DEMETER (Detection of Electro Magnetic Emissions Transmitted from Earthquake Regions) satellite which allows the detection and recognition of the natural plasma waves and facilitate retrieval of their main characteristic features such as frequency range, peak and cutoff frequencies, power intensity [6] but the detected electromagnetic emission and propagated signals are corrupted by noise and other artificial influences [7]. According to this finding such VLF signals is also embedded with noise and it is found that the average noise level in this frequency range is in the order of $0.05 \mu \mathrm{Vm}^{-1} \mathrm{~Hz}^{-1}[8]$.

Therefore before analysis of VLF data removal of noise is must. Removing the noise from the data can be considered as the process of constructing optimal estimates of the unknown signal from the available noisy data[9].There are several methods for denoising of data such as Wiener filter, short-time Fourier based methods and the latest one is wavelet shrinkage or threshold. Wiener filter technique and Short-time Fourier based methods are good for stationary signal denosing[10]. Recently wavelet transform is used for the denoising of non-stationary signals because of its good localization in time and frequency domain [11] but wavelet transform fail to denoise the signals in the non-Gaussian environments [38]. It happened because conventional signal processing techniques based on the first and second order statistics and optimized only Gaussian noise but degrade their performances in non-Gaussian environments. Higher Order Statistics (HOS) is a powerful tool for characterizing and modeling non-Gaussian noise and studies shows that VLF signals are non-Gaussian and nonstationary in nature [12], so for the denoising of VLF signals in this work we have used Independent component analysis (ICA) which is involving higher order statistics [35]for denoising the VLF signals. Independent component analysis has pronounced for the blind source separation problems (BSS) [35]. During the last few years, Independent Component Analysis (ICA) techniques have gained special attention and development [13], 
[14]. ICA is introduced in the early 1980s; it is an entropy based method[15] and introduced to separate mixtures of the neurophysiologic signals[16].ICA was originally developed to deal with problems that are closely related to the cocktail-party problem[35].The main aim of this method is to find out the unknown independent source signals that have been linearly mixed in the medium by using $4^{\text {th }}$-order statistical properties[17].These mixed signals work as the input of ICA algorithm[18].On the other side the correlation based algorithms such as principal component analysis (PCA)based on second order statistics but ICA not only applies second-order statistics but reduces the higher -order dependencies with the aim of making the signals statistically independent[18],[19].The statistical method on which ICA is based is the probability distributions and the cumulants of the mixtures ICA techniques have been widely used in feature extraction problems[20],[21],[39]source separation and denosing. Many scientists used ICA for denoising of the EEG signal[22]HRV signals[23], Speech signals[24]and functional optical imaging signal[38].Study of seismic signal is also done by ICA[15][25],[26],[27].The purpose of this paper is to study the VLF signal after denoising by ICA and evaluate the performance and computational complexity of ICA for VLF signals.

\section{Theoretical Frame work}

For the brief mathematics explanation of the ICA let us suppose that $\boldsymbol{n}$ different signals

$$
y=\left[y_{1}, y_{2}, y_{3} \ldots y_{n}\right]^{\prime} \text {. }
$$

Now hypothesize that the $\boldsymbol{n}$ signals are linear mixture of $\boldsymbol{m}$ mutually statistically independent unknown signals

$$
s=\left[s_{1}, s_{2}, s_{3} \ldots s_{m}\right]^{\prime} \text {. }
$$

Components of $\mathbf{s}$ must be non-Gaussian and this mixing is done by an unknown $\boldsymbol{n} x \boldsymbol{m}$ matrix $\boldsymbol{A}$. This mixing is essentially due to different position of satellite, path, instrumental transfer function etc. If the mixing has to be linear, nothing is assumed with respect to the source signals, which can be generated by linear or nonlinear dynamic system[28].Formally the mixture of the source signals is modeled by

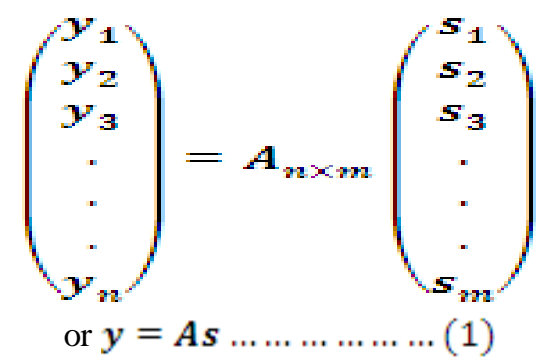

The statistical model in Eq. 1 is called ICA model[29]. Where $\boldsymbol{A}$ represent an $\boldsymbol{n x m}$ mixing matrix. For solving the ICA, $\boldsymbol{A}$ must be non-singular or full column rank when $\mathrm{m}=\mathrm{n}$ or $\mathrm{m}>\mathrm{n}$, respectively. Here we supposing that $\boldsymbol{m}$ is equal to the $\boldsymbol{n} . \boldsymbol{y}$ is an observed $\boldsymbol{n}$-dimensional vector and $\boldsymbol{s}$ is an $\boldsymbol{m}$-dimensional random vector whose components are mutually independent. The aim of ICA is to calculate the separating matrix $\boldsymbol{W}=\boldsymbol{A}^{-1}$ in such a way that the $\boldsymbol{x}=\boldsymbol{W} \boldsymbol{y}$ is an estimate $\boldsymbol{x} \sim \boldsymbol{s}$ of the original independent signals.

The properties of the ICA model is as follows [19]:

1. The independent components must be non-Gaussian for the ICA to be possible.

2. The number of linear mixed signals must be at least as large as the number of source signals.

3. The mix matrix A must be full column rank.

4. Variances (energies) of the independent components are not determined by the ICA.

5. We can not determine the order of the independent components

The basic principles of ICA estimation based on the Central limit theorem (CLT), according to that under certain condition, distribution of a sum of non-Gaussian random variables tends toward a Gaussian distribution [35]. If two or more two variables have Gaussian distribution they are independent and uncorrelated. Hence the main aim of ICA model is to maximize the non-Gaussianity to extract the independent component. The good measures of non-Gaussianity is given by the kurtosis or by the negentropy which are defined as

$$
\begin{array}{cl}
\operatorname{kurt}(x)=E\left\{x^{4}\right\}-3\left(E\left\{x^{2}\right\}\right)^{2} & \text { (Kurtosis) } \\
J(x)=H\left(x_{\text {gauss }}\right)-\boldsymbol{H}(x) & \text { (Negentropy) }
\end{array}
$$

Where $\mathbf{E}\{\}$ denote the expectation of $\boldsymbol{x} . \boldsymbol{H}(\boldsymbol{x})$ and $\boldsymbol{x}_{\text {gaus }}$ are differential entropy and Gaussian random vector of the same mean and covariance as $\boldsymbol{x}$ respectively. Several algorithm were proposed for ICA by [30],[31] ,[32].In this work we used fixed point algorithm -FastICA[29] and Mean field ICA[33]both .Firstly we focus on FastICA, following steps are engage for sources separation in this-

- Frist we make the $y$ a zero-mean variable by subtract its mean vector this process is known as centering represented as

$$
m=E\{y\}
$$


- Now by the linearly transform of observed vector $\boldsymbol{y}$ we obtain a new vector $\boldsymbol{y} \square$ which is white, i.e. its components are uncorrelated and their variances are equal to unity $(\boldsymbol{E}\{\boldsymbol{y} \boldsymbol{y}\}=\boldsymbol{I})$. This can be done by linearly multiplying $\boldsymbol{y}$ with some matrix $\boldsymbol{V}$

$$
z=V y
$$

so that we obtain a new vector $z$ that is white. It is called Whitening

- After these two processes we choose the initial unit vector $\boldsymbol{w}$ such that the projection of $\boldsymbol{w}^{T} z$ maximizes the non-Gaussianity of the single estimated source $s$.

- In this algorithm the $w$ is finding as $w \leftarrow E\left\{z g\left(w^{T} z\right)\right\}-E\left\{g^{\prime}\left(w^{T} z\right)\right\} w, w \leftarrow w /\|w\|$ where $g$ is defined as $g$ $(y)=\tanh (y)$ and $g^{\prime}(y)$ is derivative with respect to $y$

The above method is applicable only in the case of noise less ICA. If independent component are noise corrupted then noisy ICA model can be expressed by

$$
\boldsymbol{y}=\boldsymbol{A s}+\boldsymbol{n}
$$

where $n=\left(\boldsymbol{n}_{1}, \boldsymbol{n}_{2} \ldots \ldots \boldsymbol{n}_{m}\right)$ the noise vector .If noise is one of the independent component then denosing is done by basic ICA but denosing the independent components(ICs) is done by MAP(maximum a posterior) or by sparse code shrinkage[35].We are using Mean Field ICA(MFICA)[33]which is based on the MAP algorithm for denosing the ICs. In this algorithm sources are estimated by their posterior mean while maximum a posterior are used for the mixing matrix $(\boldsymbol{A})$ and noise covariance $(\Sigma)$ as

$$
\begin{aligned}
& A_{\text {MAP }}=\underset{\boldsymbol{a r g m a x}}{A} P\left(A \mid X_{y} \Sigma\right) \\
& \Sigma_{\text {ML }}=\arg \operatorname{Imax}_{\Sigma} P\left(A \mid X_{y} \Sigma\right)
\end{aligned}
$$

The above procedures are derived from expectation maximization (EM)-type algorithm. The expectation step is solved by using different mean field approaches such as variational, linear response and adaptive TAP. The MF theories produce estimates of posterior source correlations of increasing quality which is needed for maximization step for the estimate the mixing matrix and the noise[33]. A thorough description of the theory of the method is given in Ref.[33].ICA based MAP denoising in capable to denoise the non-Gaussian signals corrupted by a Gaussian/non-Gaussian noise[24].

For the evaluation of performance of this method statistical and efficiency test are conducted. Statistical testing of proposed algorithm is done by SNR (signal to noise ratio) of original and denoised signal are computed. Denoising is successful when post SNR if higher than the pre SNR [36]. It is defined as the ratio of signal power to noise power of corresponding signal.

$$
S N R=10 \log _{10} \frac{P_{\text {signal }}}{P_{\text {noise }}}=P_{\text {signal }}-P_{\text {noise }}(\text { indb) }
$$

The computation efficiency of this algorithm is computed by the mean square error (MSE) which measures the amount by which the estimator differs from the quantity to be estimated. The mathematical formula of MSE defines as [22]:

$$
M S E=\frac{1}{M N} \sum_{y=1}^{M} \sum_{x=1}^{N}\left[I(x, y)-I^{(x y y)}\right]^{2}
$$

For a perfect fit, $\mathrm{I}(\mathrm{x}, \mathrm{y})=\mathrm{I}(\mathrm{x}, \mathrm{y})$ and $\mathrm{MSE}=0$; so, the MSE index ranges from 0 to infinity, with 0 corresponding to the ideal[22].

\section{Result and Discussion}

For the study of VLF signals by ICA we have considered three electric field VLF signals observed by ICE (Instriment Champ Electrique) payload of the French Micro Satellite DEMETER launched on June 29, 2004 of different latitude and longitude, within the band width between $2 \mathrm{kHz}$ to $20 \mathrm{kHz}$ and sampling frequency $40 \mathrm{kHz}$. The signals had the length of 16384 samples. As we point out in section 2 for the work with ICA the signal must be non-Gaussians for which we computed kurtosis value of these three signals, that are given in table(1) which shows that these signals are super Gaussian(non-Gaussian) which indicate that ICA is expected to work with VLF signals. The basic model of ICA needs several mixtures of independent components (ICs) as input signals. If number of sensors/channels is more than one their signals are adopted as instantaneous mixtures like ECG signals, fMRI signals and EEG signals. In the case of single sensor an alternative way is to consider as input matrix in the mixture of many signals which are reproduced by same process. This has been done by many scientists [15],[25], [27].These signals are mixed by $3 \times 3$ random matrixes whose elements are chosen from uniformly distributed random numbers within 0 to 1 .Simulated signals are shown in figure (1) in which the original source signals are represented by $\mathrm{S} 1, \mathrm{~S} 2, \mathrm{~S} 3$ and mixture of these signals are denoted by $\mathrm{Y} 1, \mathrm{Y} 2, \mathrm{Y} 3$. In 
our simulation VLF signals are previously corrupted by noise (from the sensor) therefore adding of noise elements in these signals is not required.
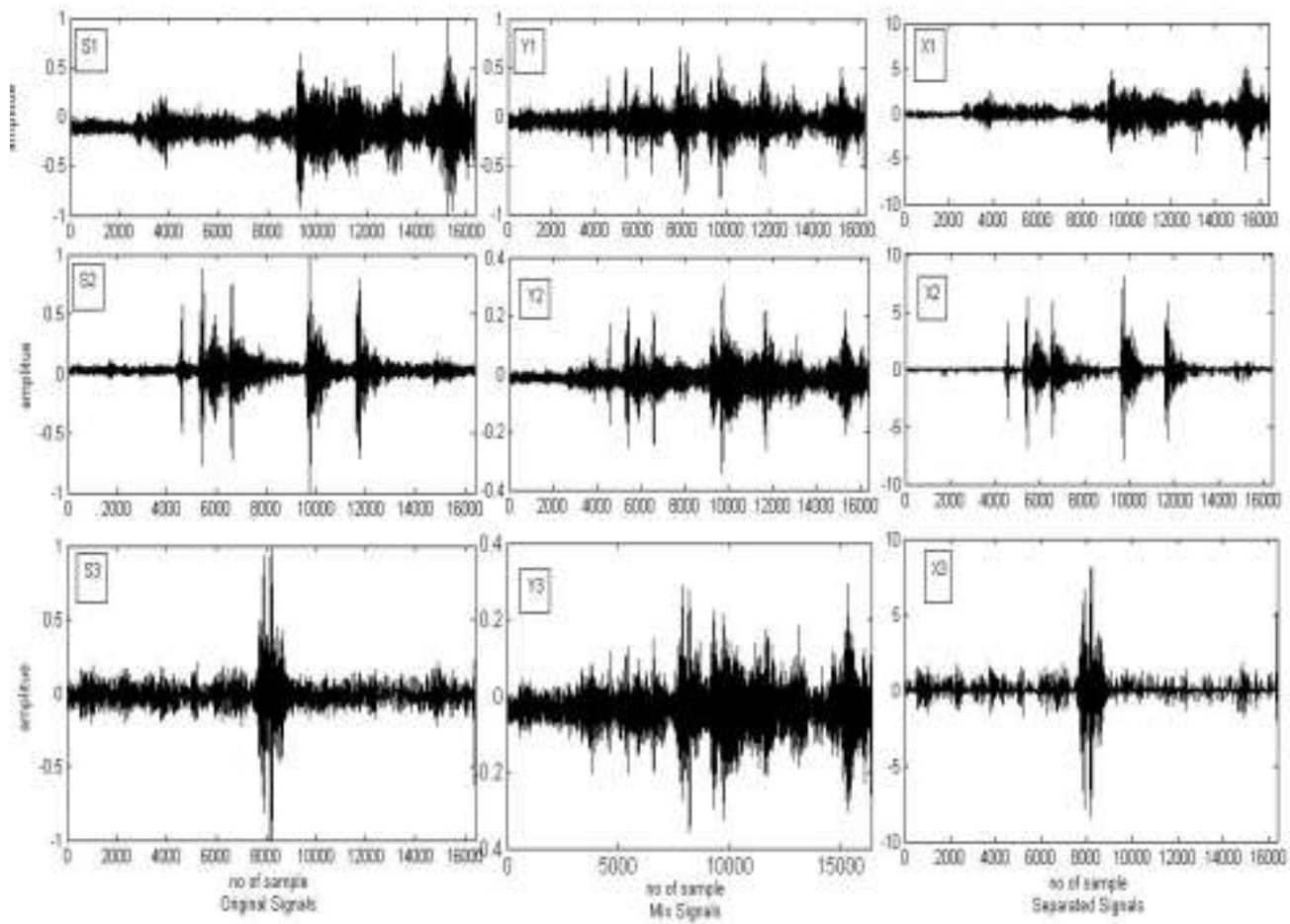

Figure1. The original signals, their mixture and separation results by ICA

In proposed method the mixture of VLF signals have been preprocessed by FastICA (www.research.ics.aalto.fi/ica/fastica) and whitening signals were calculated. These white signals are fed as input in MFICA (www.isp.imm.dtu.dk/toolbox/ica) for denosing. The output of MFICA is the clean ICs. In this way we are getting noise free Independent components from the simulated mixture of VLF signals. Figure (1) shows separated and reconstructed signals $(\mathrm{X} 1, \mathrm{X} 2, \mathrm{X} 3)$ estimated by the proposed method. It is clear from figure (1) that used ICA algorithm retained the full shape of the VLF signals and removed unwanted noise as well as increased the amplitude of reconstructed signal. It is more clear by the power spectrum density curve (PSD) shown in figure (2) that the shape of both PSD curve (with and without ICA) are same only the amplitude of all frequencies amplified .Not only that after the ICA treatment we found some sharp variation in the PSD curve that cannot observed in the original signals PSD curves.
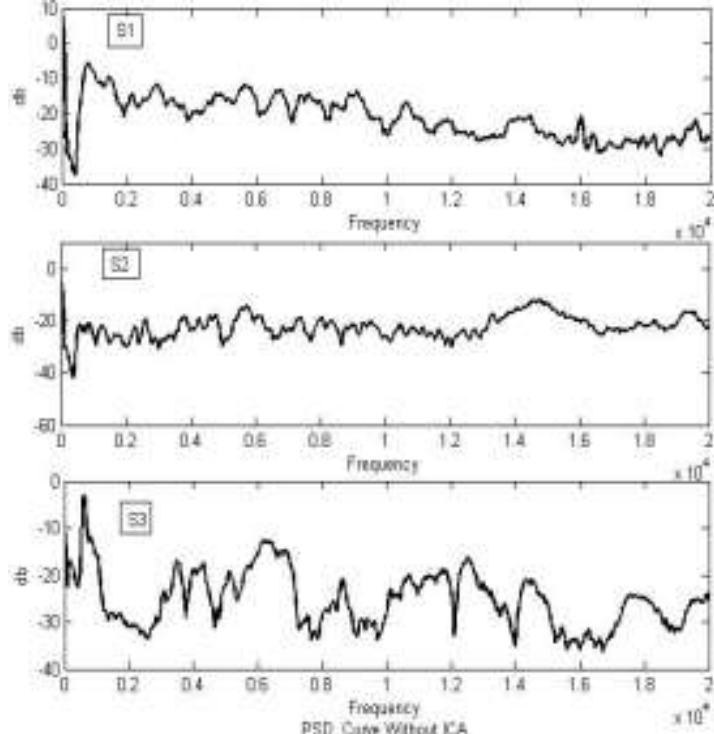
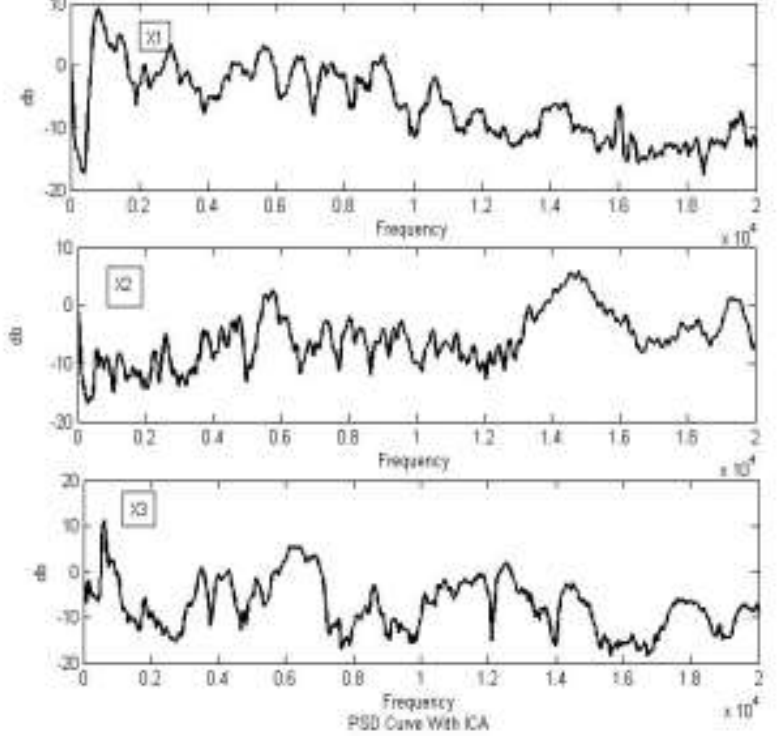

Figure2. The power spectrum density curve without ICA and with ICA of VLF signals 
In proposed method the mixture VLF signals have been preprocessed by FastICA (www.research.ics.aalto.fi/ica/fastica) and whitening signals were calculated. These white signals are feed as input in MFICA (www.isp.imm.dtu.dk/toolbox/ica) for denosing. The output of MFICA is the clean ICs. In this way we are getting noise free Independent components from the simulated mixture of VLF signals.

Figure (1) shows separated and reconstructed signals(X1, X2, X3) estimated by the proposed method. It is clear from figure (1) that used ICA algorithm retained the full shape of the VLF signals and remove unwanted noise as well as increase the amplitude of reconstructed signal. It is more clear by the power spectrum density curve (PSD) shown in figure (2) that the shape of both PSD curve (with and without ICA) are same only the amplitude of all frequencies amplified. Not only that after the ICA treatment we found some sharp variation in the PSD curve that cannot be observed in the original signals PSD curves.
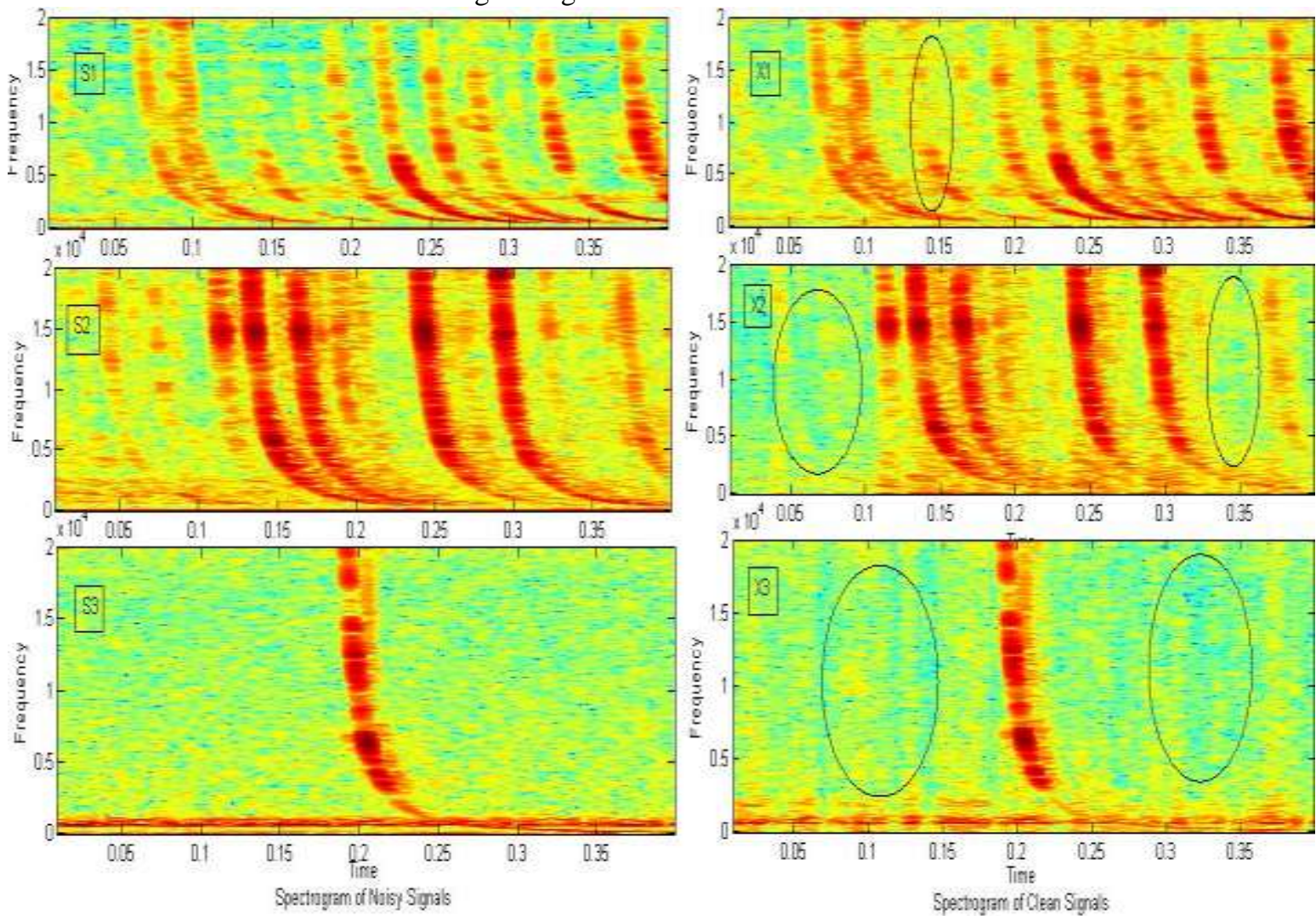

Figure3. The Spectrogram without ICA (right side) and with ICA (left side) of VLF signals (encircle area represents the enhancement and denoising).frequency in $\mathrm{kHz}$ and time in second.

Spectrograms play an important role in the study of VLF signals[40]because the fine structure of the whistlers and the precise measurements of successive echoes in whistler echo -trains were important for study of the propagation characteristics of whistler this can explore the quantitative information of exosphere that is only possible by satisfactory spectrograms[37]. Therefore we plotted spectrograms of original and reconstructed VLF signals that are shown in figure (3). It shows that after the treatment of our ICA denoising algorithm some fine 'structures are visualized and unwanted structures which are generated due to the non-Gaussian noise disappeared from the spectrogram of the reconstructed signals. One of the interesting view in the last spectrogram for signal X3 was that only the background noise is disappeared but the back side structure of whistler (double structure) does not change this indicates that this whistler is non-ducted because double trace indicates are whistler non-ducted and magnetospherically reflected near the satellite [34].Sometime this trace in generated due to noise which leads to misconceptions about the non-ducted whistlers.

\begin{tabular}{|c|c|c|c|c|}
\hline \multirow{2}{*}{ Signals } & \multirow{2}{*}{ kurtosis } & \multicolumn{2}{|c|}{ SNR(in db) } & \multirow{2}{*}{ MSE } \\
\cline { 3 - 4 } & & $\begin{array}{c}\text { Without } \\
\text { ICA }\end{array}$ & $\begin{array}{c}\text { with } \\
\text { ICA }\end{array}$ & \\
\hline S1 & 7.6 & $13 . .5$ & 15.05 & 0.75 \\
\hline S2 & 22.4 & 9.3 & 11.12 & 0.57 \\
\hline S3 & 24 & 15.3 & 16.9 & 0.55 \\
\hline
\end{tabular}

Table (1). Computed Kurtosis of VLF signals and Performance of ICA 

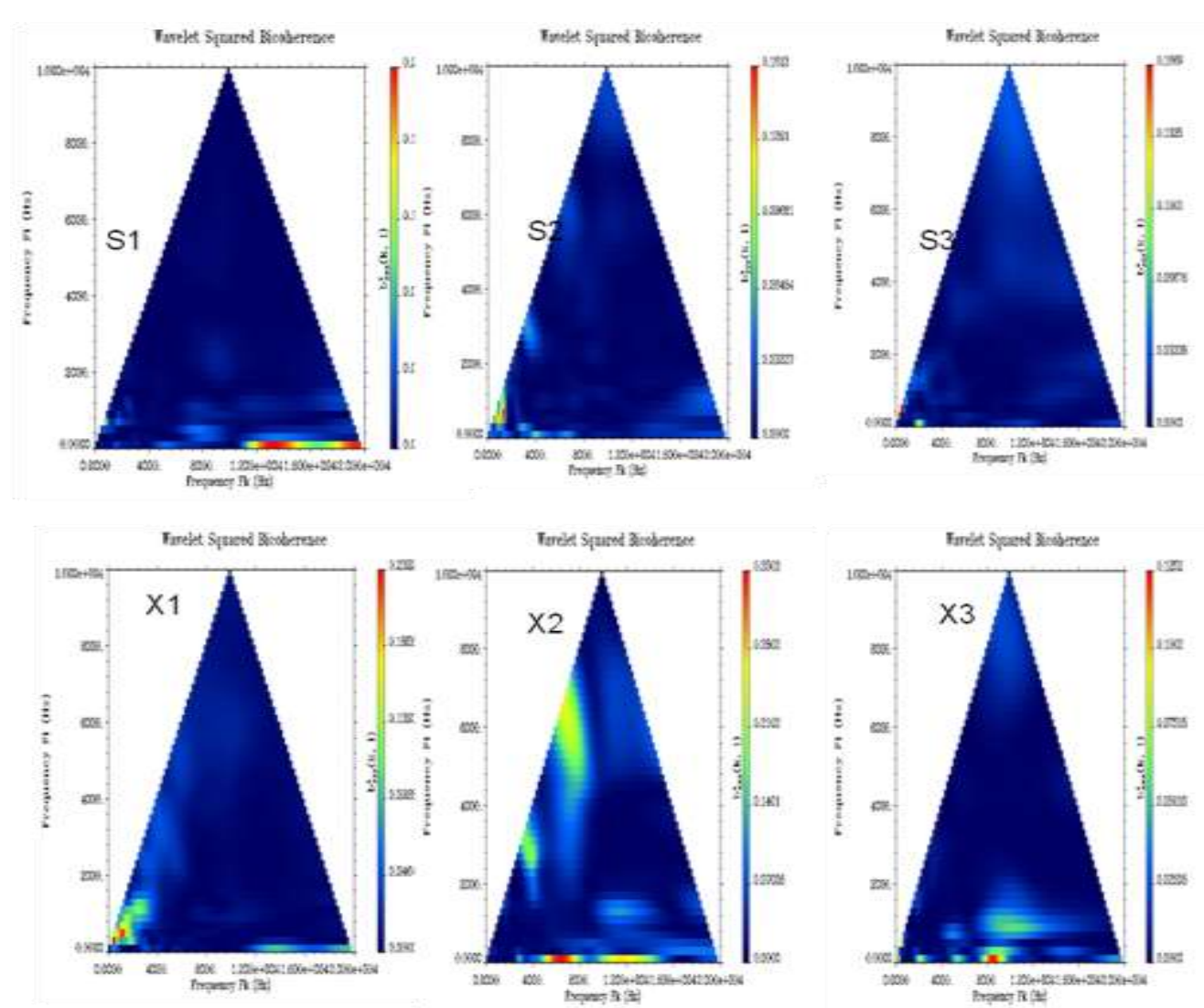

Figure 4. The wavelet bicoherence analysis without ICA (upper panel) and with ICA (lower panel)

The bicoherence analysis is used for the study of nonlinear interaction and quadratic phase coupling between two frequencies or waves. Spectrum broadening in VLF due to nonlinear interaction between the VLF and Extremely Low frequency (ELF) is analysis by bicoherence[41].In order to compare the efficiency of ICA preprocess, wavelet based bicoherence is directly applied to the VLF signals, without ICA preprocess and result is plotted in figure (4) (upper plane) and with ICA preprocess plotted in lower plane .Comparing both we are getting more peaks in higher frequency band in bicoherence maps after the ICA preprocessing which indicates that ICA increase the non-Gaussianity of the signals. These results give better understanding for the study of nonlinear interaction between higher frequencies band in VLF signals which are impossible in original signals.

High value of signal to noise ratio (SNR) is the indication of good quality of signal.VLF signals are very weak signals therefore for the study of VLF signals improvement in SNR is very important [37].So for that we treated consider signals with proposed method and calculate the SNR of this signals. After and before treatment by proposed ICA ,calculated SNR of VLF is given in table (1).We found that SNR of VLF signals increase with ICA preprocess that indicated toward the improvement in quality of this signals. The efficiency evaluation of proposed algorithm for VLF signals is computed by MSE and we found that the value of MSE for these signals is very small due to which we retain the original shape considering the VLF signals.

\section{Conclusion}

In this paper, we have presented a new approach of ICA for denoising the VLF signals. We use two different ICA algorithms, FastICA and MFICA simultaneously. For the source (signals) separation, process of whitening has obtained by FastICA while maximum a posteriori estimates are used for the mixing matrix and the noise covariance. The analysis result shows that the proposed method is most efficient for the retrieval of the non-Gaussian/Gaussian noise from the VLF signals and able to separate the non-stationary VLF signals.

To observe the results of proposed ICA algorithm based denosing of VLF signals we plotted spectrogram and wavelet bicoherence map. We found that it improve the sharpness of spectrograms and enhanced results of the wavelet bicoherence maps in high frequency band of VLF signals. The high SNR conclude that it is an efficient technique for improving the quality of VLF signals. The lower values of MSE focus on less distortions in the reconstructed signals. Thus we concluded that statistical method based ICA algorithm is a robust tool for gaining better understanding of VLF signals.

In our study we have consider the different type of VLF whistler signals. We will also continue the method with other VLF signals such as hiss, chorus, tweeks etc. One of the promising study shows that the VLF 
spectra are computed onboard of DEMETER every $51.2 \mathrm{~ms}$ and cover the frequency range from $19.53 \mathrm{~Hz}$ to 20 $\mathrm{kHz}$ in 1024 channels evenly spaced by $19.53 \mathrm{~Hz}$ and in ICE payload observed VLF data noise present due to the parasite signals and it is increased toward the lower frequency level[6]. On the base of this study we will be further develop a method in which we combine wavelet transform with ICA to decomposed the signal is many frequency level and denoise at each level

\section{Acknowledgement}

One of us (Shivali Verma)is grateful to MPCST for financial support through JRF.

\section{References}

[1] R. Barra , D. Llanwyn Jonesb, C.J. Rodgerc ELF and VLF radio waves Journal of Atmospheric and Solar-Terrestrial Physics 62 (2000) 1689-1718.

[2] Morris B. Cohen, Umran S. Inan, and Evans W. Paschal "Sensitive Broadband ELF/VLF Radio ReceptionWith the AWESOME Instrument" IEEE TRANSACTIONS ON GEOSCIENCE AND REMOTE SENSING, VOL. 48, NO. 1, JANUARY 2010 pp3-17

[3] Masashi Hayakawa, "VLF/LF Radio Sounding of Ionospheric Perturbations Associated with Earthquakes" Sensors 2007, 7, 11411158 .

[4] A. Rozhnoi, M. Solovieva1, O. Molchanov, O. Akentieva, J. J. Berthelier, M. Parrot, P. F. Biagi, andM. Hayakawa, "Statistical correlation of spectral broadening in VLF transmitter signal and low-frequency ionospheric turbulence from observation on DEMETER satellite" Nat. Hazards Earth Syst. Sci., 8, 1105-1111, 2008.

[5] Cesidio Bianchi and Antonio Meloni, "Natural and man-made terrestrial electromagnetic noise: an outlook" ANNALS OF GEOPHYSICS, VOL. 50, N. 3, June 2007.

[6] T. Onishi , J.J Berthelier Latmos, "Synthetic characterization of VLF electric field spectra from the ICE experiment on DEMETER Automatic Recognition and Characterization of natural and man made emissions", Notes of IPSL Planetary Group, ISSN 17680042, June 2010.

[7] Hanna Rothkaehl,Nataly Izohkina,Igor Prutensky,Sergey, "17 Ionospheric disturbances generated by different natural processes and by human activity in Earth plasma environment", Annals of Geophysics, Vol. 47, N. 2/3, 2004,pp.1215-1225.

[8] J.J Berthelier,M.Godefroy ,F.Leblanc,M Malinger ,M.Menvielle.D. Lagoutte, J.Y.Brochot,F. Colin,F. Elie, C.Legendre, C. Legendre,P.Zamora, D.Benoist ,Y Chapuis,J Artru,R.Pfaff, “ICE,the electric field experiment on DEMETER” ,Planetary and Space Science April 2006,pp.456-471.

[9] Imola K. Fodor and Chandrika Kamath, 2003. "Denoising Through Wavelet Shrinkage: An Empirical Study", Journal of Electronic Imaging, Vol. 12, pp.151-160.

[10] Galvao, Roberto Kawakami Harrop "Comparative study on instrumental signal denoising using Fourier and Wavelet transforms", Quím. Nova [online]. 2001, Vol.24, pp. 874-884.

[11] M. Sifuzzaman1, M.R. Islam1 and M.Z. Ali, "Application of Wavelet Transform and its Advantages Compared to Fourier Transform," Journal of Physical Sciences 2009, Vol. 13, pp. 121-134.

[12] F.Elie,M. Hayakawa ,M.Parrot, J. Pincon ,F. Lefeuvre "Neural Network System for the Analysis of transient phenomena on board the DEMETER micro-satellite", IEICE TRANS FUNDAMENTALS,vol. E82-A,no. 8 Aug, 1999.

[13] C Jutten and J Herault , “ Blind separation of sources, part I: An adaptive algorithm based on neuromimetic architecture.” Signal Processing, 1991, 24, pp.1-10.

[14] P. Comon, "Independent component analysis: a new concept" Signal Process36 (3) (1994) 287-314

[15] E. De Lauro, S. De Martino, M. Falanga, and M. Palo, "Self-sustained vibrations in volcanic areas extracted by Independent Component Analysis: a review and new results" Nonlin. Processes Geophys., 18, 925-940, 2011.

[16] J.H'erault and B. Ans,' 'R'eseaux de neurons 'a synapses modifiables:D'ecodage de messages sensoriels composites par une apprentisagenon supervise et permanent" C.R. Acad. Sci. Paris III,525-528, 1984

[17] C. Puntonet," New algorithms of source separation in linear media" Ph.D. thesis,Department of Architecture andTechnology of Computers, University of Granada, Spain,1994.

[18] S. Cruces, L. Castedo, A. Cichocki, "Robust blind source separation algorithms using cumulants," Neurocomputing 49 (2002) 87-18.

[19] A. Hyvärinen, E. Oja, "Independent Components Analysis:A Tutorial," Laboratory of Computer and Information Science, Helsinki University of Technology, 1999.

[20] P. Hoyer and A. Hyv"arinen, "Independent component analysis applied to feature extraction from colour and stereo images" Network: Computation in Neural Systems, 2000, 11(3), pp191-210, 2000

[21] Jie Hao, Xin Zou, Martin P. Wilson, Nigel P. Davies, Yu Sun,Andrew C. Peet and heodoros N. Arvanitis, "A comparative study of feature extraction and blind source separation of independent component analysis (ICA) on childhood brain tumour $1 \mathrm{H}$ magnetic resonance spectra", Research Article NMR Biomed Wiley InterScience. 2009, 22,pp. 1-10 .

[22] Janett Walters-Williams \& Yan Li, “A New Approach to Denoising EEG Signals - Mer" International Journal of Biometrics and Bioinformatics Volume (5) : Issue (2) : 2011,pp.130-148

[23] FJ Gimeno-Blanes, JL Rojo-Álvarez, J Requena-Carrión2, E Everss,J Hernández-Ortega, F Alonso-Atienza, A García-Alberola, “ Denoising of Heart Rate Variability Signals During Tilt Test Using Independent Component Analysis and Multidimensional Recordings", Computers in Cardiology 2007,34,pp.399-402.

[24] Xin Zou, Peter Jan covic, Ju Liu and M"unevver K"ok"uer, "ICA-BASED MAP ALGORITHM FOR SPEECH SIGNAL ENHANCEMENT", ICASSP 2007, IEEE, 2007, pp569-572

[25] J.J. Gonzalez de la Rosa, C.G. Puntonet, I. Lloret, "An application of the independent component analysis to monitor acoustic emission signals generated by termite activity in wood" Measurement ELSESVIER, 37 (2005),pp. 63-76.

[26] Acernese, F., Ciaramella, A., De Martino, S., De Rosa, R., Falanga,M., and Tagliaferri, R.: Neural networks for blind-source separationof Stromboli explosion quakes, IEEE Trans. Neural Networks14, 167-175, 2003.

[27] Acernese, F., Ciaramella, A., De Martino, S., Falanga, M., Godano,C., and Tagliaferri, R.: Polarisation analysis of the independent components of low frequency events at Stromboli volcano (EolianIslands, Italy), J. Volcanol. Geotherm. Res., 137, 153-168, 2004.

[28] De Lauro, E., De Martino, S., Falanga, M., and Palo, M.: Statistical analysis of Stromboli VLP tremor in the band [0.1-0.5] Hz:some consequences for vibrating structures, Nonlin. Processes Geophys., 13, 393-400, 2006.

[29] Hyv"arinen A, Oja E. 2000. "Independent component analysis:algorithms and applications." Neural Netw 13: 411-430.

[30] Bell, AJ \& Sejnowski, TJ. "An information maximization approach to blind separation and blind deconvolution" Neural Computation 7, 1129-1159, 1995. 
[31] Hyvarinen, A , “A family of fixed-point algorithm for independent component analysis." In Proc.IEEE Int. Conf. on Acoustics Speech and signal Processing (ICASSP'97), pages 3917-3920, Munich, Germany, 1997.

[32] J.F.Cardoso,"High-order contrasts for independent component analysis". NeuralComputation 11, 157-192,1999.

[33] P. A. d. F. R. Hojen-Sorensen, O. Winther, and L. K. Hansen, "Mean-Field Approaches to Independent Component Analysis," Neural Computation, vol. 14, pp. 889-918, 2002.

[34] R. L.Smith and J. J. Angerami, "Magnetospheric properties deduced from OGO 1 observations of ducted and nonducted whistlers" J. Geophys.Res., 73, 1, 1968

[35] Hyvarinen A, Karhunen J, Oja E. Independent Component Analysis. John Wiley \& Sons, Inc.: Chichester, 2001.

[36] Stephane Mallat,"A wavelet tour of signal Processing,third Edition” Academic Press, 1998.

[37] Roger M. Gallet, "The Very Low-Frequency Emissions Generated in the Earth's Exosphere", Proceedings of the I.R.E, 1959, February, pp.211-231.

[38] Uzay E.Emir,Ceyhun B.Akgul,Ata Akin ,Aysin Ertuzun,Bulent Sankur,Kerem Harmanci, "Wavelet denoising vs Ica denosing for functional optical imaging”,Neural Engineering ,2003, conference Proc.,International IEEE, march 2003,pp.384-387.

[39] J. Hurri, A. Hyv"arinen, J. Karhunen, and E. Oja "Image feature extraction using independent component analysis" In Proc. NORSIG'96, Espoo, Finland, 1996p.p 475-478.

[40] David Shklyar, "Numerical simulation of spacebron VLF spectrograms related to lighting-induced emissions" Proc.of ISSS-7, March 2005, pp.26-31.

[41] Satoru Ohnami and Masashi Hayakawa "Bispectrum analysis of nonlinear interaction between VLF transmitter signals and ELF emissions" proceedings of ISAP'92,SAPPORO,Japan pp no 197-200 